\begin{abstract}
> Comment les médecins intègrent-ils à leur pratique clinique, jusqu'alors axée sur l'anatomie pathologique, la découverte des micro-organismes et la théorie des germes? Portent-ils un nouveau « regard » sur le malade et son corps infecté ? Comment «traquer» ces microbes? Comment les détruire? Loin d'une « révolution pastorienne »foudroyante, ces questions nous plongent dans une histoire prudente, laborieuse, parfois dramatique, qui inscrit le laboratoire dans la clinique et intègre la science dans la médecine. <
\end{abstract}

L'infectiologie, de dénomination récente (ce néologisme désigne la spécialité médicale des maladies infectieuses), constitue aujourd'hui une discipline à part entière de la médecine. Or, quand l'historien met en regard deux classifications des maladies établies en France par des cliniciens, celle d'Augustin Grisolle, parue en 1862 [1], et celle de Charles Bouchard, publiée en 1903 [2], une différence majeure saute aux yeux: c'est l'apparition, chez Bouchard, des «maladies infectieuses », classe d'entités morbides à part entière.

Plusieurs aspects restaient à explorer sur cette question. En France, les travaux historiques ont surtout porté sur les aspects théoriques et ont plutôt privilégié le rôle de Louis Pasteur. La plupart des historiens ou sociologues français qui ont souscrit à une analyse de la «révolution pastorienne » et de la «pasteurisation » de la médecine (notamment Jacques Léonard, Claire Salomon-Bayet, Bruno Latour) délimitent leurs approches aux aspects «franco-français »: Pasteur et les hygiénistes français, importance des « hommesrelais » du pastorisme ou des « réseaux » pastoriens [38]. Ces travaux introduisent une certaine rupture dans I'historiographie. Toutefois, ils conservent à Pasteur un

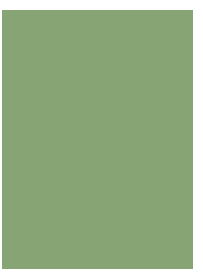

Centre de Recherches médecine, sciences, santé et société,

rôle central dans la mesure où celui-ci contrôle le « réseau » qu'il met lui182, boulevard de La Villette, 75019 Paris,

France. dire les « hommes-relais», intermédiaires entre son laboratoire, l'hygiène, la clinique et la société. Mais on apprend peu sur ces cliniciens au quotidien, et cette façon de considérer les médecins surtout comme des « importateurs » des idées pastoriennes, conduit peutêtre à négliger le fait que ces praticiens font eux-mêmes du laboratoire, d'une manière d'ailleurs différente de celle de Pasteur, qu'ils travaillent sur l'étiologie, qu'ils se saisissent des outils de la bactériologie et bâtissent leur propre bactériologie clinique, en traitant de leurs propres problèmes et expériences de médecin. Dans son analyse de ce qu'il appelle la «pasteurisation de la médecine », Latour met surtout en avant l'efficacité technique des sérums. Or ce qui touche la pratique clinique des maladies infectieuses s'articule avant tout autour du diagnostic, il y a intégration des outils bactériologiques à une forme modifiée de l'anatomie pathologique.

De plus, les études historiques françaises ont également eu tendance à sous-estimer l'importance d'une bactériologie médicale et d'une clinique des maladies infectieuses qu'on pourrait dire européenne, en particulier allemande. En effet, dès lors que la bactériologie de Robert Koch s'est constituée par rapport à des questions de cliniciens, n'y a-t-il pas lieu de penser qu'elle s'ajuste plus immédiatement à la démarche anatomo-clinique que les recherches de Pasteur et de ses disciples, plus axées sur l'immunité et la prévention? Autrement dit, la bactériolo- 
gie des médecins, répondant aux exigences de la clinique, n'a-t-elle pas été au fond plus proche de celle qui s'élaborait en Allemagne que de Pasteur en ces temps de nationalisme exacerbé où la mise en avant de notre « génie national » avait valeur de renaissance de la patrie après le désastre de 1870 [9]?

En matière de thérapeutique, même constat. Les travaux publiés portent surtout sur les traitements par sérums et la prévention par les vaccins $[10,11]$, et l'on fait en règle générale remonter les débuts de la « chimiothérapie » à 1906, date de la découverte du Salvarsan par Paul Ehrlich en Allemagne [12]. Du même coup, les historiens ont porté peu d'attention aux transformations de la thérapeutique médicale contemporaines de la construction et du développement de la théorie des germes. Cette lacune n'exprimerait-elle pas une adhésion implicite au lieu commun médical moderne, selon lequel «l'art » du clinicien serait très éloigné de cette «science de laboratoire » qu'est la bactériologie, affaire de spécialistes maîtrisant des techniques spécifiques qui, en évoluant, deviendraient étrangères à la majorité des praticiens? Lieu commun qui se voit démenti dès lors que l'on y regarde de plus près. En effet, ce qui est remarquable dans les années 1870 1880 , c'est précisément que clinique et bactériologie médicale se construisent l'une en référence à l'autre, s'enrichissant mutuellement.

\section{Recomposition nosographique}

Vers 1860 en France, avant l'émergence de la « théorie des germes », le travail clinique est guidé par un schéma de pensée lié à la démarche anatomo-clinique fondée sur le rapport entre lésions et symptômes, entre les altérations et lésions constatées sur le cadavre à l'amphithéâtre, et les symptômes et signes observés au cours de la maladie et notés au lit du malade. Avec l'observation, la palpation, la percussion et l'auscultation, il est possible d'identifier des « anomalies » anatomiques sur le malade vivant $[13,14]$. De nombreuses maladies « infectieuses » et « contagieuses » sont décrites selon ces critères et classées dans les « empoisonnements septiques », les « sécrétions morbides », les «fièvres », les «inflammations », etc. [1].

L'émergence de la théorie des germes, vers 1870 1880 , ne bouleverse pas tout; elle remet en perspective les définitions anatomo-cliniques des maladies; elle rencontre des entités cliniques constituées, elle les réorganise et les réunifie autour d'une perspective étiologique. Les « miasmes », «virus » et autres particules invisibles étaient des concepts utilisés depuis longtemps. Les « petites granulations », « ferments » ou
« infusoires », observés de plus en plus fréquemment au microscope au sein de certains tissus, sont désormais considérés comme des « germes » vivants qui jouent un rôle dans les fermentations et dans certaines maladies. Mais pour de nombreux cliniciens de cette époque, le problème étiologique semble être l'un des plus difficiles et des plus obscurs de la pathologie. Dans un certain nombre de maladies «virulentes spécifiques » (comme la variole par exemple), l'agent causal se dérobe en effet à l'observation microscopique.

Une doctrine pathogénique tend cependant à s'imposer: les maladies spécifiques sont dues à l'action d'un agent, un « être parasitaire », un « ferment animé », vibrion ou bactérie, qui entre dans l'organisme et s'y multiplie. Tant que cette multiplication dure, elle provoque des troubles fonctionnels symptomatiques, et il y a alors maladie infectieuse. L'idée que ces germes vivants peuvent être impliqués dans certaines maladies conduit à des remaniements, à des modifications dans la manière d'interpréter les entités cliniques existantes, et tout un travail étranger à l'esprit des pastoriens. Travail de recomposition des catégories, travail classique de la médecine (fondé cette fois sur l'étiologie qui devient une question centrale). Pendant cette période de la fin du XIXe siècle, des pans entiers de la pathologie sont à nouveau nosographiés, parallèlement au développement d'une bactériologie médicale naissante.

Les médecins donc, ne font pas du pastorisme, ils font de la clinique. Pourquoi, dans cette recomposition nosographique basée sur l'étiologie, pourquoi Koch plutôt que Pasteur? Parce que c'est essentiellement l'école allemande qui fournit les moyens d'identifier les bactéries et de faire le diagnostic in vivo. Dans les années 1880, des cliniciens hospitaliers parisiens tels Sigismond Jaccoud, Georges Dieulafoy, Louis Landouzy ou Charles Bouchard, grands lecteurs des recherches cliniques publiées outre-Rhin en même temps que des travaux écrits en France, cherchent à lier leurs pratiques médicales antérieures à une nouvelle approche de l'étiologie des maladies infectieuses et contagieuses. La recomposition nosographique va constituer une aide au diagnostic. La recherche quasi-systématique des germes dans les différentes maladies prend d'ailleurs peu à peu en défaut l'axiome un germe - une maladie. En effet, à la conception d'entités morbides causées par des microbes spécifiques et toujours les mêmes, il faut en ajouter une autre, moins «simple», à savoir que des processus morbides en apparence identiques, au moins dans leurs manifestations premières (telles les angines, méningites, endocardites, bronchites, etc.), peuvent être provoqués par des microbes différents ou par l'association de plusieurs microbes. 
Au début du XXe siècle, la classe des «maladies infectieuses » apparaît; elles sont devenues une classe d'entités morbides à part entière. Bouchard réunit des maladies qui jusque-là avait été séparées et rangées dans des classes diverses [2]. II sépare les «maladies infectieuses » en deux sous-groupes, selon que l'agent étiologique est «spécifique » ou «non spécifique » (les maladies sont elles-mêmes distinguées selon la nature du microorganisme: parasite, champignon, bactérie, ou origine inconnue). Ce classement, très proche de celui d'aujourd'hui, n'a été possible que grâce au développement concomitant des techniques microbiologiques et des pratiques cliniques.

\section{Diagnostic}

La pathologie infectieuse ne s'est pas construite uniquement autour de la bactériologie; la réorganisation a été plus globale et plus complexe, en continuité avec des connaissances anatomo-cliniques établies depuis longtemps. Aux signes, symptômes et lésions repérables sur le malade vivant, les cliniciens associent un germe. II y a élaboration progressive d'une sémiologie clinique des maladies infectieuses, évolution de la démarche diagnostique, mise au point de techniques de prélèvement, de «traque du germe » (ce qui implique des outils et des gestes nouveaux) et tout cela, parallèlement au développement des techniques bactériologiques d'isolement et de culture des germes.

Des études minutieuses des courbes de température sont par exemple entreprises [15]. Les médecins en tirent des renseignements précieux en matière de diagnostic et de pronostic. L'examen de la courbe de température, associé aux autres points d'appel cliniques, fait rechercher des germes particuliers dans certains liquides ou tissus. La pratique clinique s'attache à la recherche systématique des signes qui accompagnent la «fièvre »: frissons, élévation des fréquences cardiaque et respiratoire, sueurs, augmentation de la taille de la rate, aspect de la langue, ganglions, etc. L'observation clinique, la comparaison des « processus morbides », la prise en compte des conditions de leur apparition (leur histoire naturelle, leur symptomatologie) sont les éléments à partir desquels les médecins présument la nature infectieuse d'une maladie. La bactériologie y ajoute un élément de certitude ou de forte probabilité diagnostique.

À travers les itinéraires d'un certain nombre de cliniciens parisiens, on peut mettre en évidence comment à la fin du XIXe siècle, l'idée de soumettre le diagnostic clinique au «verdict » de l'examen bactériologique prend progressivement consistance: cette nouvel- le manière d'appréhender la maladie modifie la pratique clinique et le rapport au malade et à son corps: celui-ci est « habité » de germes microscopiques qui circulent et se multiplient. Le mal invisible, venu de l'extérieur, peut se développer dans des fluides essentiels du corps et ronger sournoisement l'individu. La maladie semble considérée comme une présence intempestive, ennemie, étrangère au malade, entrée par effraction, et qu'il faut combattre.

II s'agit alors de rechercher les germes en effectuant au lit des malades des prélèvements de gorge, de crachats, de sang, de liquide vertébral, pleural, etc., avec lesquels on ensemence aseptiquement des milieux de culture. C'est l'époque de la mise au point par exemple de la «culture du sang », qu'on appellera plus tard hémoculture [16], qui implique d'ailleurs des innovations techniques (inventer, par exemple, une seringue entièrement stérilisable), mise au point également de la ponction «vertébrale » ou ponction lombaire [17]. Ces techniques de prélèvements, effectués aseptiquement au lit du malade par les médecins cliniciens, permettent une recherche bactériologique immédiate.

Ces nouveaux gestes et ces nouveaux outils, dirigés vers la traque du germe sont liés à la représentation de la maladie infectieuse: sous la peau du malade, dans tel ou tel organe, des collections purulentes se forment, des abcès se collectent, le mal sournois se développe. II s'agit de repérer ce mal et de l'extirper, séparer l'infecteur de l'infecté, le coupable de la victime, l'étranger du «soi-même ». On est vraiment dans une problématique médicale. De même qu'il y a des techniques d'observation des lésions, il y a des techniques de mise en évidence de l'agent causal. Nouvelles conceptions, nouveaux gestes, nouveau matériel, nouveau rapport entre clinique et petit laboratoire installé de façon plus ou moins improvisée au sein même du service hospitalier. C'est une véritable «mutation anthropologique » du clinicien que la théorie des germes et le développement de la bactériologie médicale ont entraînée.

Ce laboratoire n'est pas le laboratoire pastorien, mais le laboratoire d'analyse diagnostique. Là encore, les outils utilisés sont surtout ceux de la bactériologie médicale allemande: Koch propose l'utilisation des milieux solides ce qui permet d'obtenir facilement des cultures pures de bactéries; il met au point des outils particuliers pour le travail sur ces milieux: anse et fil de platine, etc. Mais ce qui marque particulièrement les cliniciens français, c'est sa découverte du bacille de la tuberculose en 1882 [18]. Bouchard et Dieulafoy poussent d'ailleurs les internes de leurs services à rechercher le bacille dans les crachats des malades, dès 1883 . L'examen des crachats et la recherche du bacille de la 
tuberculose rend également de grands services aux médecins de ville. La présence du bacille permet au praticien d'affirmer précocement qu'il y a tuberculose pulmonaire. Si ce microbe est retrouvé, cela conduit à une prescription précoce de l'isolement, évitant peut être la transmission du germe à d'autres membres de la famille.

Dans les cas d'angine, il est affirmé que les signes cliniques n'ont qu'une valeur illusoire du point de vue diagnostique, et que seul l'examen bactériologique permet d'identifier le microbe responsable de la maladie: bacille diphtérique, ou autres germes (streptocoque, pneumocoque). Dans le premier cas, le sérum antidiphtérique est administré aussitôt (à partir de 1894). Dans l'autre, un traitement antiseptique local suffit. Ces recherches du bacille tuberculeux dans les crachats, comme du bacille diphtérique dans les fausses membranes, constituent un enjeu de santé publique. De nombreuses discussions et débats ont lieu à l'Académie de médecine de Paris à propos du diagnostic et du classement nosologique des angines. Selon Dieulafoy, le vrai diagnostic, le diagnostic complet, celui qui importe au pronostic, à la prophylaxie, à la thérapeutique des angines, c'est le diagnostic qui sait accoler à l'expression symptomatologique de l'angine son expression étiologique spécifique [19]. La classification anatomo-clinique des angines non diphtériques n'est pas invalidée, mais complétée. Les différentes formes cliniques des angines ne sont pas associées à un seul agent infectieux et, réciproquement, un même germe semble pouvoir donner des formes différentes d'angine. L'observation clinique garde donc toute son importance: rougeur du pharynx, tuméfaction des amygdales, parfois œdème des piliers, aspect du voile ou de la luette, présence d'un « enduit blanc crémeux », etc. Cela permet de situer l'angine dans la classification anatomoclinique et constitue le support de la démarche diagnostique et étiologique.

Le laboratoire devient donc un auxiliaire nécessaire de la clinique et associe recherche diagnostique et expérimentale. Il est le creuset d'études diagnostiques, physiopathologiques et thérapeutiques directement en lien avec les observations cliniques faites sur les malades du service. On est loin du laboratoire de recherche fondamentale, éloigné des malades. Avec les examens bactériologiques, I'association clinique-laboratoire est particulièrement étroite. Cette association semble entraîner un large consensus à la fin du XIXe et au début du XXe siècle. Ce diagnostic bactériologique effectué au laboratoire, Dieulafoy le revendique comme une véritable « annexion à la clinique ». La clinique est couplée, associée au laboratoire, sans que celui-ci prenne l'ascendant sur celle-là.

\section{Thérapeutique}

Généralement oubliée par les travaux historiques, l'élaboration, à la fin du XIXe siècle, des bases scientifiques d'une thérapeutique chimique anti-infectieuse par les antiseptiques méritait d'être rappelée. Une des raisons possibles de cette occultation, outre la compétition avec la thérapeutique par les sérums de l'Institut Pasteur, c'est que des doutes sérieux se sont élevés, dès le début du XXe siècle, sur l'efficacité de certains antiseptiques administrés par voie générale. A l'enthousiasme réel des débuts a succédé un certain scepticisme lié à leur toxicité. Toutefois, l'efficacité de cette thérapeutique au niveau cutané et local fut incontestable. Elle est plutôt envisagée comme un acte de contre-agression en administrant une substance susceptible de juguler la cause du mal. Le « credo » des pasteuriens est plutôt de rechercher les moyens de prévenir l'infection que de la traiter, et ils délaissent l'autre voie. On constate que le modèle thérapeutique est lié à la représentation de la maladie.

Les premiers essais de «thérapeutique chimique » des maladies infectieuses se produisent à l'hôpital, en France, dans les années 1880, notamment dans le service de Bouchard à Paris [20]. II débute ses essais de traitement de la phtisie à l'aide d'un antiseptique, la créosote, à la fin des années 1870 [21]. Pour lui, la thérapeutique de la tuberculose est le principal souci des médecins de son époque. S'il multiplie les essais thérapeutiques contre cette maladie, c'est parce que selon lui, elle « décime l'humanité ». Bouchard recherche activement les antiseptiques les mieux appropriés pour lutter contre les maladies infectieuses. Si ses recherches sur les moyens d'obtenir l'antisepsie locale, l'antisepsie du tube digestif ou au sein des tissus n'ont guère abouti à la mise au point d'antiseptiques aisément maniables lors d'infections générales graves, elles mettent en lumière les principes concernant l'emploi de ces agents (principes qui paraissent très proches de ceux utilisés aujourd'hui avec les antibiotiques).

Selon Bouchard, pour juger de la valeur thérapeutique d'un antiseptique, il faut établir qu'il raccourcit la durée et combat les effets d'une maladie infectieuse. L'argumentation est bien clinique. Mais avant d'entreprendre des essais chez les malades, il précise qu'il faut évaluer pour chaque substance chimique son pouvoir antiseptique in vitro. II met au point un « test de l'équivalent antiseptique » et définit pour les germes trois concentrations: celle qui ralentit le développement microbien, celle qui arrête ce développement, celle qui tue le microbe [22]. II faut également évaluer sa toxicité chez l'animal (test de «l'équivalent toxique »), à 
des doses inoffensives pour l'homme. II n'est pas nécessaire selon lui que l'antiseptique tue le microbe, il suffit qu'il « diminue ou retarde sa pullulation »; le plus important étant que l'antiseptique ne soit pas toxique. On assiste également avec les travaux de Bouchard à la naissance d'une certaine pharmacologie clinique des antiseptiques. La thérapeutique par les antiseptiques est un « traitement de choc ». II s'agit d'un combat sans merci contre l'ennemi, parfois d'une grande brutalité. A la lecture de certaines observations médicales, on perçoit ce goût du « radical »: le but est l'extermination du germe, quelles qu'en soient les difficultés pour le malade.

Les recherches thérapeutiques, réalisées essentiellement par des cliniciens passionnés par les travaux expérimentaux, et le plus souvent effectuées dans des laboratoires intégrés aux services d'hospitalisation, semblent plus proches des travaux menés en Allemagne à cette époque que de ceux réalisés à l'Institut Pasteur. La thérapeutique chimique des maladies infectieuses par les antiseptiques s'inscrit dans une pratique médicale complexe et il n'y a pas lieu de la mettre en compétition ou en concurrence avec la sérothérapie pasteurienne. II s'agit de deux choses différentes. Mais l'histoire des sciences, volontairement ou non, renforce parfois l'idée d'une science fondamentale toute-puissante et seule porteuse de vérité. L'occultation du rôle du travail clinique dans l'invention thérapeutique, par exemple, semble assez systématique.

\section{Chaires de bactériologie et de clinique des maladies infectieuses à la faculté de médecine de Paris}

Au début du XXe siècle, la situation de la bactériologie dans l'univers hospitalier parisien est complexe. Le microbe est devenu un «objet » de savoirs, de pratiques spécifiques et d'enseignement pour différentes disciplines médicales comme l'anatomie pathologique, la pathologie expérimentale, l'hygiène, l'histoire naturelle et la thérapeutique, chacune construisant son propre « objet-microbe ». Ces disciplines médicales sont en concurrence les unes avec les autres en ce qui concerne l'enseignement de la bactériologie, et la faculté se trouve elle-même en concurrence avec l'Institut Pasteur. La «bactériologie clinique » se développe dans d'autres domaines que ceux privilégiés par les pastoriens (immunité, prévention) et trouve plutôt ses références au-delà de nos frontières auprès de l'École Allemande.

Dans un rapport critique, Charles Bouchard demande avec force en 1907 la création d'une chaire de bactériologie à la faculté de médecine de Paris. Les étudiants se plaignent, dit-il; ils voudraient trouver à la faculté et
« pas seulement à l'Institut Pasteur », un enseignement technique de bactériologie. Ils voudraient pouvoir savoir ce qui est nécessaire pour comprendre la pathologie infectieuse, devenir capables de pratiquer ce qui est indispensable pour assurer leur diagnostic et agir avec plus de sûreté en ce qui concerne le traitement de leurs malades. Bouchard estime donc absolument nécessaire la création d'une chaire de «clinique des maladies infectieuses » à côté d'une chaire de bactériologie. L'isolement dans des hôpitaux spéciaux des malades atteints de maladies infectieuses fait que les étudiants, qui ne sont pas admis auprès de ces isolés, ont du mal à acquérir la connaissance clinique et pratique de ces maladies [23].

II faut finalement attendre 1918 pour voir la création d'une chaire théorique de bactériologie associée à celle d'une chaire de clinique des maladies infectieuses. Un des freins de l'institutionnalisation de la bactériologie médicale à la faculté de médecine de Paris a été l'Institut Pasteur. Elle n'a pu se constituer qu'en relation avec l'institutionnalisation d'une clinique des maladies infectieuses établie à l'hôpital Claude Bernard. Souhaitant la prise en charge spécifique de la pathologie infectieuse, la faculté demande la création de ces deux chaires.

L'École des pastoriens, qui a tant renouvelé la biologie, s'est montrée réticente à l'égard de ce qui semblait la relativiser ou la dépasser. Deux exemples ont illustré mon propos: les débuts de la thérapeutique chimique antimicrobienne par les antiseptiques et la rivalité avec la faculté de médecine pour l'enseignement de la bactériologie. II faut souligner l'effort de certains cliniciens qui profitent de leur position et de leur prestige social pour mener à bien des réalisations qui tiennent souvent à un combat pour l'innovation, en même temps qu'à la diffusion progressive d'un savoir issu d'institutions hospitalières, généralement parisiennes. Une analyse de la constitution réciproque d'une bactériologie médicale et d'une pathologie infectieuse a été proposée. Cette «clinique des maladies infectieuses », qu'on nomme aujourd'hui « infectiologie », ne peut être considérée comme une simple application du pastorisme, comme la seule conséquence du « réseau pensant » pasteurien décrit par Bruno Latour.

Les cliniciens construisent une «pathologie infectieuse » en prenant en compte non seulement l'étude des bactéries et de leur virulence, mais aussi l'étude fine de la sémiologie clinique des infections, directement auprès des malades, la recherche diagnostique, la mise au point de techniques de prélèvement destinées à « traquer » les germes, l'installation de laboratoires directement associés aux services de médecine, la recherche thérapeutique [24]. II y a donc quelque injus- 
tice à dire que la médecine recueillait des fruits dont aucune part ne lui reviendrait, et il est difficile de concevoir la production du savoir bactériologique qui ne devrait rien aux théories médicales. $\diamond$

\section{SUMMARY}

The birth of infectiology in France

Between 1870 and 1918, the field of « infectious pathology » developped in France. It consisted of an overall reorganization; clinicians attempted to associate a « germ » with the living patient's signs, symptoms and detectable lesions. A clinical symptomatology of infectious diseases was progressively elaborated, diagnostic procedures evolved, techniques of « hunting » for the germs and anti-infectious therapy were devised in parallel with the development of microbiological techniques to isolate and culture the microorganisms. Around 1870, the germ theory was assimilated into the established anatomical-clinical approach. Etiology became a central question, albeit not a new one. During the 1880's, hospital physicians, for example G. Dieulafoy and C. Bouchard, refused to separate clinical medicine from that of the laboratory, claiming the necessity to combine the two. Based on clinical observations, doctors deduced the infectious nature of a disease; bacteriology added diagnostic and etiological elements. At the end of the 19 th century, the idea of submitting a clinical diagnosis to the « verdict » of a bacteriological test progressively took form: « hunting » for the germs bay taking samples and then inoculating them into culture medium. This concept entails lab work closely associated with the clinical diagnostic procedure. Bouchard actively sought the antiseptics the best adapted to fight against certain infectious entities. According to him, to evaluate the therapeutic value of an antiseptic, it has to be established that it shortens the duration and counters the effects of the infection. Althoug the fundamental argument is clearly clinical, it must first be proven that the medication is effective against the microbe in vitro and that it is as non-toxic as possible in animals. At the Paris School of Medicine, the field of « medical bacteriology » took form around the concept of « infectious diseases », then located at Hôpital Claude Bernard. $\diamond$

\section{RÉFÉRENCES}

1. Grisolle A. Traité de pathologie interne. Paris: Masson, 1862.

2. Bouchard C. Traité de pathologie générale. Paris: Masson, 1903.

3. Dagognet F. Méthodes et doctrines dans l'œuvre de Pasteur. Paris: PUF, 1967.

4. Salomon-Bayet C. Pasteur et la révolution pastorienne. Paris: Payot, 1986.

5. Léonard J. La médecine entre les pouvoirs et les savoirs. Paris: Aubier, 1981.

6. Murard L, Zylberman P. L'hygiène dans la République. La santé publique en France, ou l'utopie contrariée, 18701918. Paris: Fayard, 1996.

7. Latour B. Les microbes guerre et paix. Paris: A.M. Métailié, 1984.

8. Latour B. Pasteur. Paris:
Perrin, 1995.

9. Bonnah C. Instruire, guérir, servir. Formation et pratique médicale en France et en Allemagne. Strasbourg: PUS, 2000.

10. Moulin AM. Le dernier langage de la médecine. Histoire de l'immunologie de Pasteur au Sida. Paris: PUF, 1991.

11. Moulin AM. L'aventure de la vaccination. Paris: Fayard, 1996.

12. Canguilhem G. Idéologie et rationalité dans l'histoire des sciences de la vie. Paris: Vrin, 1988.

13. Contrepois A. Notes on the early history of infective endocarditis and the development of an experimental model. Clin Infect Dis 1995; 20: 461-6.

14. Contrepois A. Towards a history of infective endocarditis. Medical History 1996; 40: 25-54.
15. Contrepois A. Prise de température et maladies infectieuses, en France, à la fin du XIXe siècle. Pyrexie 1997; 1: 37-8.

16. Contrepois A. Naissance de I'hémoculture. Rev Prat 1995; 45: 942-7.

17. Contrepois A. Les méningites avant et après la théorie des germes (18601890). Pyrexie 1999; 3 : 147-8.

18. Contrepois A. Une maladie infectieuse pas comme les autres. La tuberculose à I'hôpital Laennec. In : Moulin AM, Contrepois A, eds. De l'Hostpital des Incurables à l'hôpital Laennec. Paris: Hervas, $2000: 68-81$.

19. Dieulafoy G. De l'angine diphtérique à forme herpétique. Bull Acad Med 1895; $33: 600-8$ et $34: 7-17$.
20. Contrepois A. Débuts de la thérapeutique chimique des maladies infectieuses en France. Rev Prat 2000; 50 : 1750-4.

21. Bouchard C. Notes sur l'emploi de la créosote vraie dans le traitement de la phtisie pulmonaire. Gazette Hebdomadaire 3 août 1877.

22. Bouchard C. Thérapeutique des maladies infectieuses. Paris: Savy, 1889.

23. Bouchard C. Questions relatives à la réforme des études médicales. Paris: Steinheil, 1907.

24. Contrepois A. L'invention des maladies infectieuses. Naissance de la clinique des maladies infectieuses en France. Paris: Éditions des Archives Contemporaine, 2001 